Article

\title{
Band Gap Tuning in 2D Layered Materials by Angular Rotation
}

\author{
Javier Polanco-Gonzalez ${ }^{1}$, Jesús Alfredo Carranco-Rodríguez ${ }^{2}$, José L. Enríquez-Carrejo ${ }^{1}$, \\ Pierre G. Mani-Gonzalez ${ }^{1}$, José Manuel Domínguez-Esquivel ${ }^{3}$ and Manuel $\operatorname{Ramos}^{1 \text {,* }}$ \\ 1 Departamento de Física y Matemáticas, Instituto de Ingeniería y Tecnología, \\ Universidad Autónoma de Cd. Juárez, Avenida del Charro \#450 N. Cd. Juárez, Chihuahua C.P. 32310, \\ Mexico; javier.polanco@uacj.mx (J.P.-G.); jose.enriquez@uacj.mx (J.L.E.-C.); pierre.mani@uacj.mx (P.G.M.-G.) \\ 2 Materials Science and Engineering, McMaster University, 1280 Main St. West, Hamilton, \\ ON L8S4L7, Canada; carrancj@mcmaster.ca \\ 3 Instituto Mexicano del Petróleo, Eje Central Lázaro Cárdenas No 152, México D.F. 07730, Mexico; \\ jmdoming@imp.mx \\ * Correspondence: manuel.ramos@uacj.mx; Tel.: +52-656-688-6909; Fax: +52-656-688-4813 \\ Academic Editor: Jung Ho Je \\ Received: 23 October 2016; Accepted: 11 January 2017; Published: 8 February 2017
}

\begin{abstract}
We present a series of computer-assisted high-resolution transmission electron (HRTEM) simulations to determine Moiré patters by induced twisting effects between slabs at rotational angles of $3^{\circ}, 5^{\circ}, 8^{\circ}$, and $16^{\circ}$, for molybdenum disulfide, graphene, tungsten disulfide, and tungsten selenide layered materials. In order to investigate the electronic structure, a series of numerical simulations using density functional methods (DFT) methods was completed using Cambridge serial total energy package (CASTEP) with a generalized gradient approximation to determine both the band structure and density of states on honeycomb-like new superlattices. Our results indicated metallic transitions when the rotation approached $8^{\circ}$ with respect to each other laminates for most of the two-dimensional systems that were analyzed.
\end{abstract}

Keywords: Moiré patterns; $\mathrm{MoS}_{2} ;$ graphene; $\mathrm{WS}_{2} ; \mathrm{WSe}_{2} ; \mathrm{HRTEM}$

\section{Introduction}

Layered materials have attracted much attention lately, because of their exceptional catalytic, photovoltaic and semiconducting properties [1,2]. An extensive characterization for 2D materials such as molybdenum disulfide $\left(\mathrm{MoS}_{2}\right)$, tungsten disulfide $\left(\mathrm{WS}_{2}\right)$, tungsten diselenide $\left(\mathrm{WSe}_{2}\right)$ and graphene has occurred in the past decades, using field emission gun microscopic techniques, such as scanning electron [3], transmission electron [4] and tunneling microscopy [5] with high accuracy. Observations reveal in detail their layered sandwich structure consisting of S-Mo(W)-S and Se-W-Se layers held together at a distance of $0.62 \mathrm{~nm}$ by weak Van der Waals forces [6]. The formation of Moiré patterns in those particular layered materials was observed by Kobayashi using scanning tunneling microscopy [5], and by Yacaman implementing the transmission electron microscopy technique [7], both concluding that the formation of Moiré patterns is attributed to strain effects between layered slabs with respect to the (001) crystallographic direction, creating honeycomb-like structures. Additionally, Jasinski observed similar Moiré patterns for single layers of graphene attributed to a $5^{\circ}$ of rotation between slabs [8]. Recently, we were able to determine the electronic band structure of strained $2 \mathrm{R}-\mathrm{MoS}_{2}\left(\mathrm{SG}-\mathrm{R} 3 \mathrm{~m}\right.$ ) when it is rotated about $10^{\circ}$ (with respect to the (001)-basal plane). Our density functional theory (DFT) calculations determined a rapid semiconducting-to-metallic state transition, as indicated by two irreducible points near $\mathrm{K} \rightarrow \Gamma$ and $\mathrm{A} \rightarrow \mathrm{L}$ when sampling over the Brillouin zone with Fermi wave vector values of $k_{F}$ at $\sim 0.47 \AA^{-1}$ and $k_{F}$ at $\sim 0.37 \AA^{-1}$ near $10^{\circ}$ to $12^{\circ}$ of rotation; for 
more details, we invite the reader to review in detail our manuscript as indicated in Reference [9]. However, the point we would like to introduce here is the formation of a new lattice parameter, which is a new O-lattice formed by the rotation of crystallographic structures as described by Bollman in late 1970s [10] and presented by Remskar [11] and Gomez and Romeu for the reciprocal lattice rotation between two crystals [12]. Creating this natural strain between slabs is relatively easy due to the weak Van der Waals bonding between the slabs, and this is the reason why it was possible to create a single layer of graphene as presented by Geim and Novoselov et al. [13]. Zhang et al. were able to achieve tuning of single layers of the graphene band gap when fabricating a field-effect transistor [14]; thus, we present a series of new O-lattices for honeycomb-like structures as formed by rotating 2D layered structures with respect to the (001) basal plane, along with a series of computer-assisted electronic structure calculations using density functional methods to identify if there is any transition (tuning) from semiconducting to metallic and vice versa on those specific nanostructure materials.

\section{Results and Discussion}

Typical Moiré patterns are observed when two 2D lattices are rotated at a certain angle, and this observation is made directly at their basal plane, usually called (001), over the $c$-axis direction. We reported these observations previously in experimental HRTEM and simulated them for 2R-MoS 2 slabs when rotated about $12^{\circ}$ in the $c$-direction [3,9]. Furthermore, we were able to produce the formation of Moiré patterns on other materials (i.e., tungsten disulfide $\left(\mathrm{WS}_{2}\right)$, graphene and tungsten diselenide $\left(\mathrm{WSe}_{2}\right)$ ) by achieving this lattice rotation on the molecular models, which created honeycomb-like features, observing as well the formation of new O-lattices as presented in Figure 1 (corresponding to the distance between ending points at the edge of the honeycomb-like structure). Later, those molecular models were subjected to HRTEM simulations, varying $a, b$ coefficients in the projected potential as described by Equation (1) in Section 3.1. The as-simulated sequence of images is presented in Figures 2-5; it is possible to determine the formation of a large O-lattice with a non-defined honeycomb structure for angles of rotation corresponding to $3^{\circ}$ and a strong honeycomb structure at $16^{\circ}$. Moreover, the resulting O-lattice was measured digitally using the BIOVIA ${ }^{\circledR}$ Materials Studio crystal builder tool. Our findings are presented (for easy reading) in Table 1 and graphically by the inset of red arrows in Figure 1.
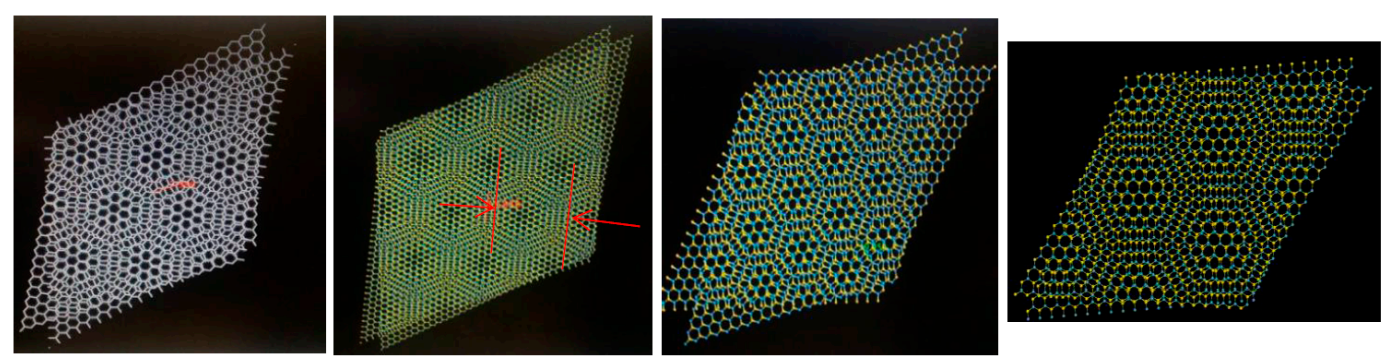

Figure 1. Moiré pattern as formed by angular rotation of 2D layered materials molecular models. From left to right: graphene, tungsten diselenide $\left(\mathrm{WSe}_{2}\right)$, tungsten disulfide $\left(\mathrm{WS}_{2}\right)$ and molybdenum disulfide $\left(\mathrm{MoS}_{2}\right)$ at $19^{\circ}$ of rotation.

It is important to mention that Moire patterns are not typically seen by HRTEM techniques for bulk 2D-layered materials; for example, when surveying $2 \mathrm{R}-\mathrm{MoS}_{2}$ slabs, fringes are commonly observed and it is possible to determine the Van der Waals force gap of $6.2 \AA$ between slabs [8] and also to see clearly that synthesis conditions can cause the "turbostraticity" bending of the layered structure. Thus, one must have cautious procedures when fabricating nanoscale-organized devices [9]. Therefore, analysis of the electronic structure corresponding to rotated 2D layered structures needs to be performed. The electronic structures of these O-lattices, as calculated by DFT methods, reflect a rapid transition for the majority of these layered structures; our findings are presented in Figure 3. 


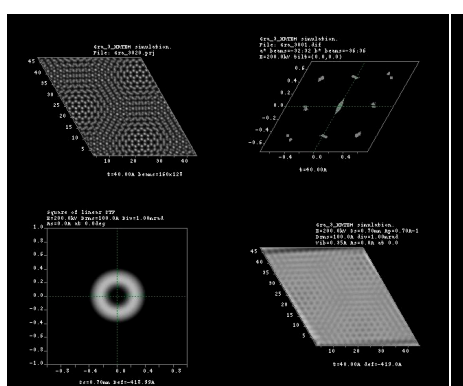

(a)

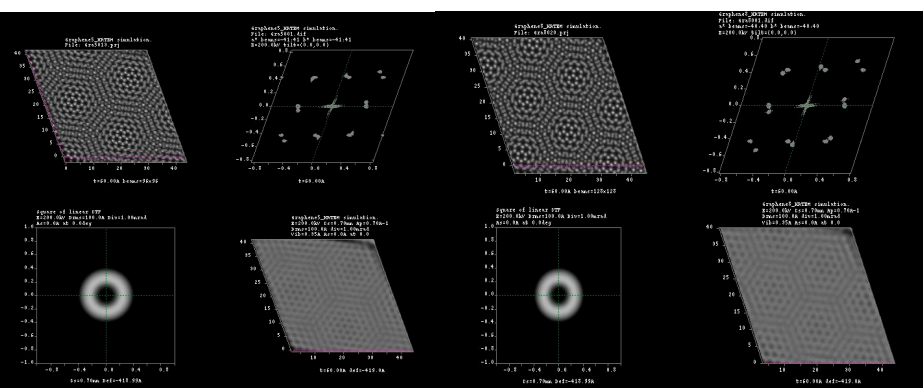

(b)

(c)

Figure 2. HRTEM simulation for (a) $3^{\circ}$, (b) $8^{\circ}$ and (c) $16^{\circ}$ of rotation in graphene molecular structure.

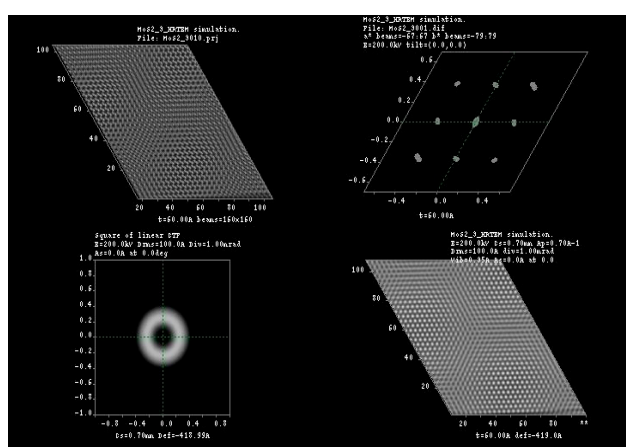

(a)

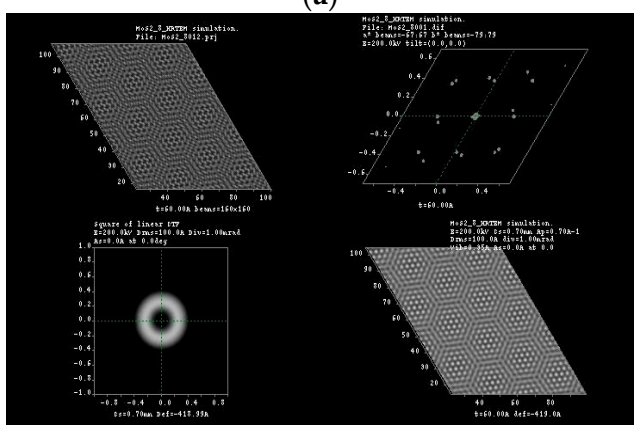

(c)

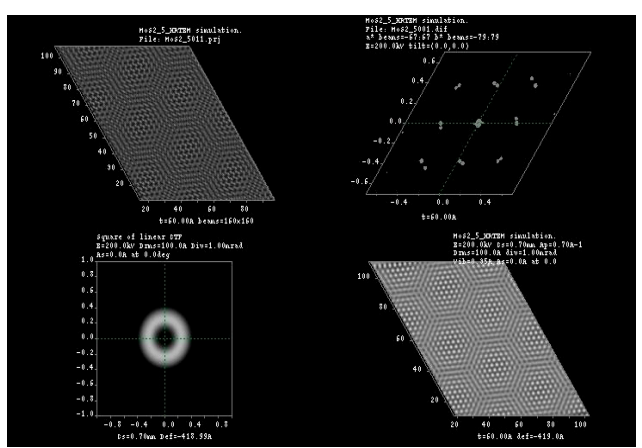

(b)

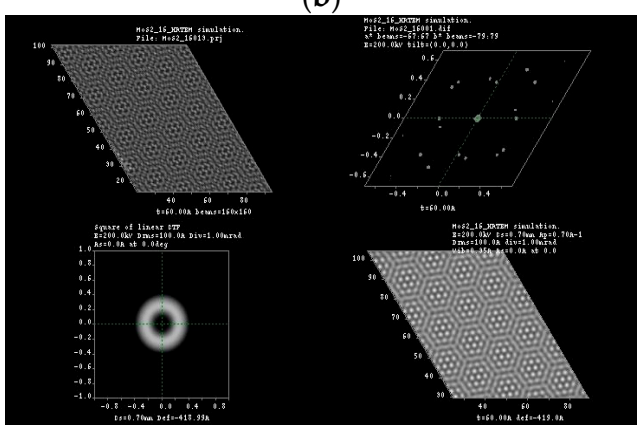

(d)

Figure 3. HRTEM simulation for (a) $3^{\circ}$; (b) $5^{\circ}$; (c) $8^{\circ}$ and (d) $16^{\circ}$ of rotation in molybdenum disulfide molecular structure.

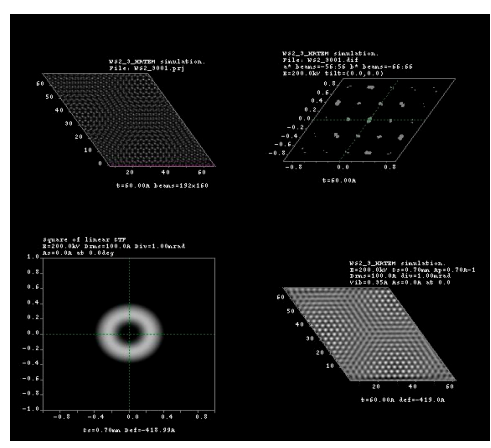

(a)

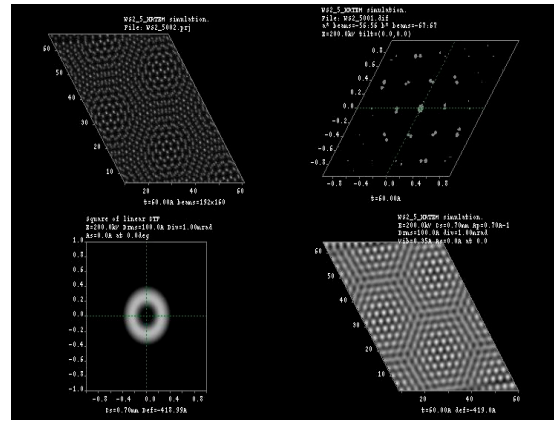

(b)

Figure 4. Cont. 


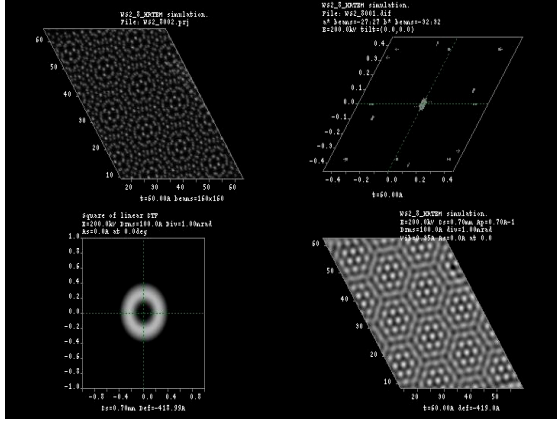

(c)

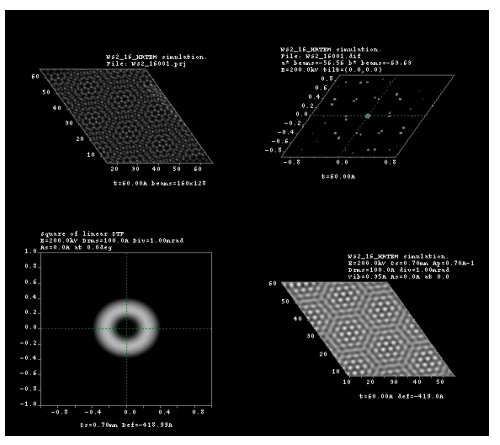

(d)

Figure 4. HRTEM simulation for $(\mathbf{a}) 3^{\circ} ;$ (b) $5^{\circ}$; (c) $8^{\circ}$ and (d) $16^{\circ}$ of rotation in tungsten disulfide molecular structure.

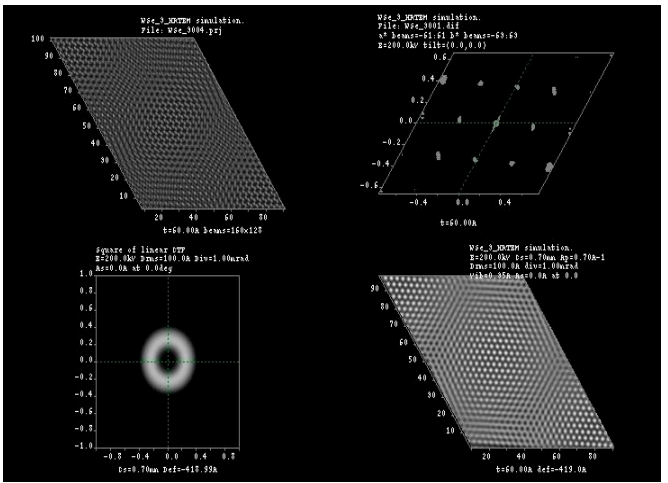

(a)

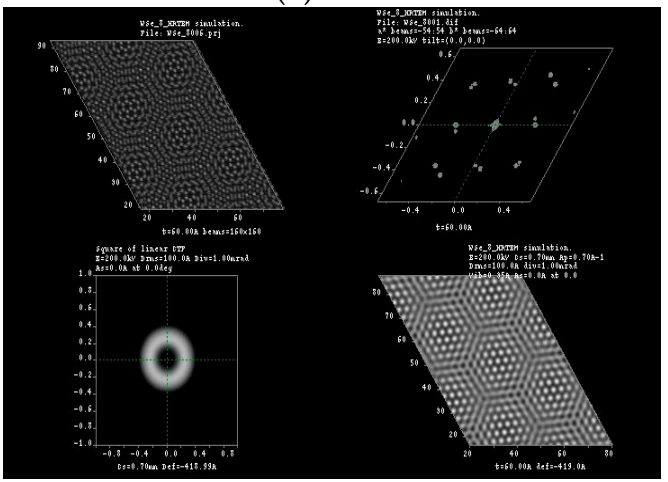

(c)

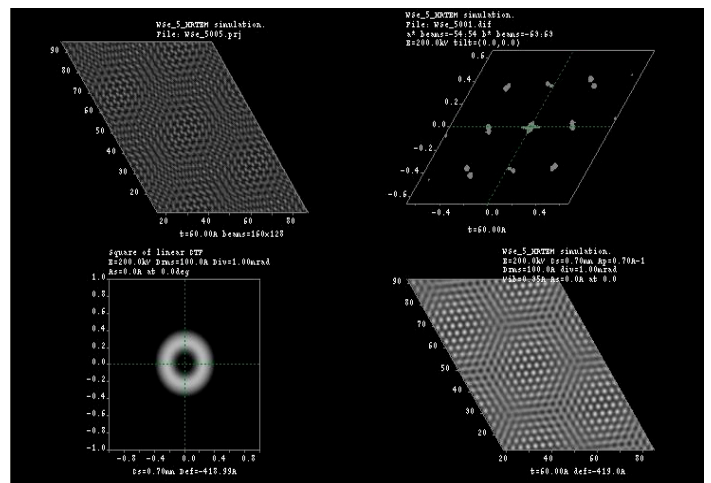

(b)

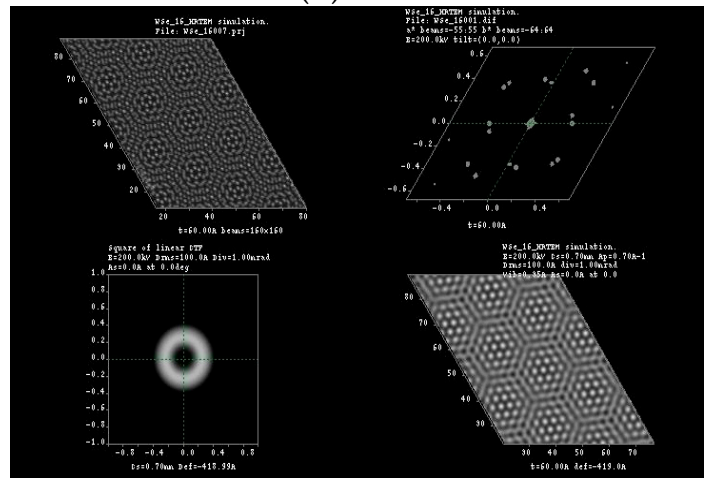

(d)

Figure 5. HRTEM simulation for (a) $3^{\circ}$; (b) $5^{\circ}$; (c) $8^{\circ}$ and (d) $16^{\circ}$ of rotation in tungsten diselenide molecular structure.

Table 1. O-lattice distribution for $2 \mathrm{D}$ layered materials corresponding to molecular structures at various rotation angles.

\begin{tabular}{ccccc}
\hline Rotation Angle & Graphene & MoS $_{\mathbf{2}}$ & WSe $_{\mathbf{2}}$ & WS $_{\mathbf{2}}$ \\
\hline $3^{\circ}$ & $12.300 \AA$ & $25.328 \AA$ & $22.974 \AA$ & $18.948 \AA$ \\
$5^{\circ}$ & $9.840 \AA$ & $15.830 \AA$ & $19.692 \AA$ & $15.796 \AA$ \\
$8^{\circ}$ & $8.639 \AA$ & $15.736 \AA$ & $14.899 \AA$ & $9.950 \AA$ \\
$16^{\circ}$ & $7.380 \AA$ & $12.725 \AA$ & $9.846 \AA$ & $9.474 \AA$ \\
\hline
\end{tabular}

In order to determine the electronic structure of those new O-lattices as created by the angular rotation of the molecular structures, we proceeded with band structures using CASTEP, as described 
in Section 3.2 and elsewhere [9]. Our results indicated some transitions from metallic into a wide-band-gap semiconductor for the graphene layers, as presented by the band structure and density of states corresponding to $0^{\circ}$ and $16^{\circ}$ shown in Figure 6 . The opposite occurs for the case of the $\mathrm{WS}_{2}$ and $\mathrm{WSe}_{2}$ and $\mathrm{MoS}_{2}$ (presented in Reference [9]) molecular structures, as presented in Figures 7 and 8.
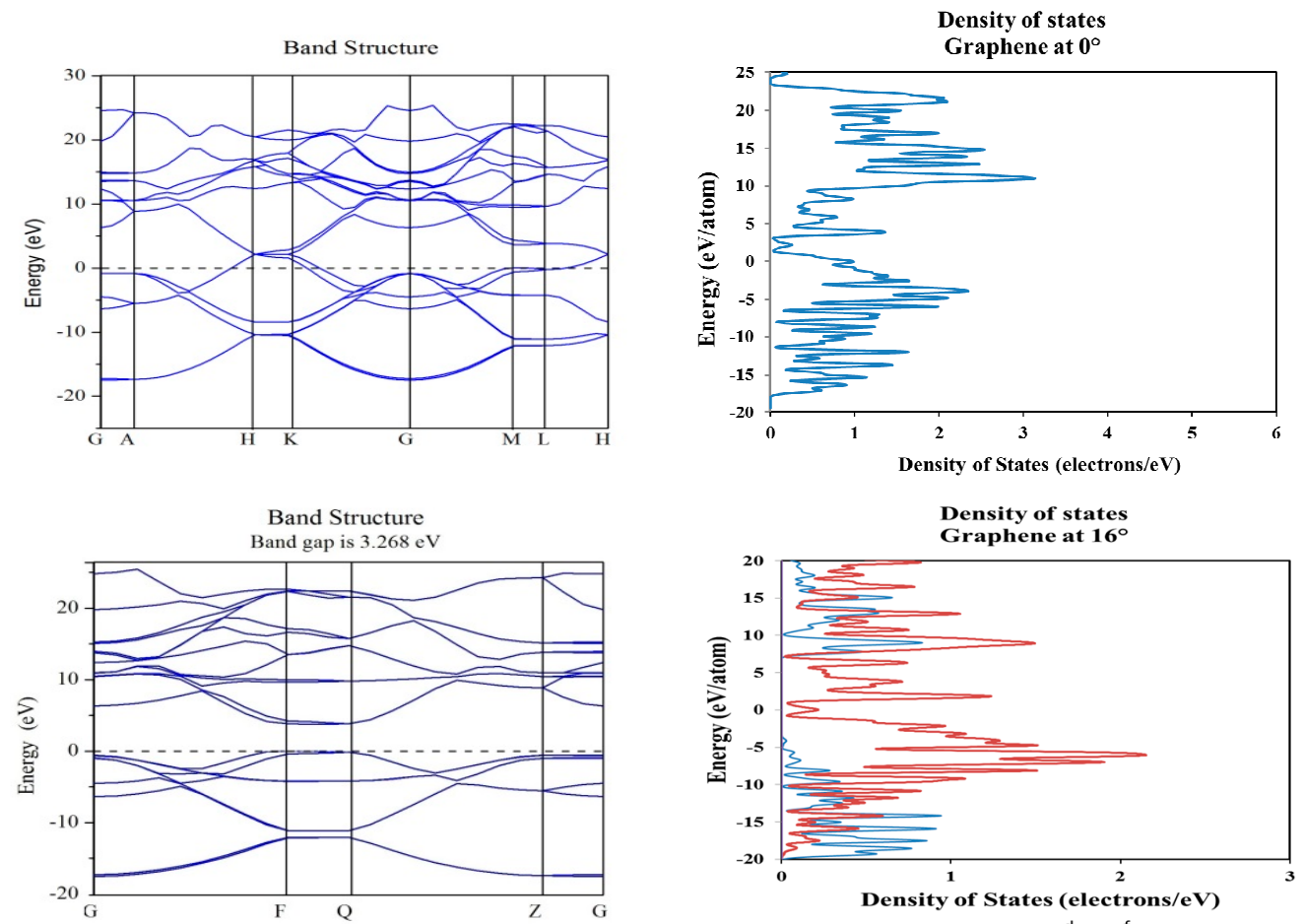

Figure 6. Band structure and density of states plots corresponding to $0^{\circ}$ (top) and $16^{\circ}$ (bottom) of rotation in graphene molecular structure; a tuning on the electronic band gap is observed near the Fermi level $(0 \mathrm{eV})$.
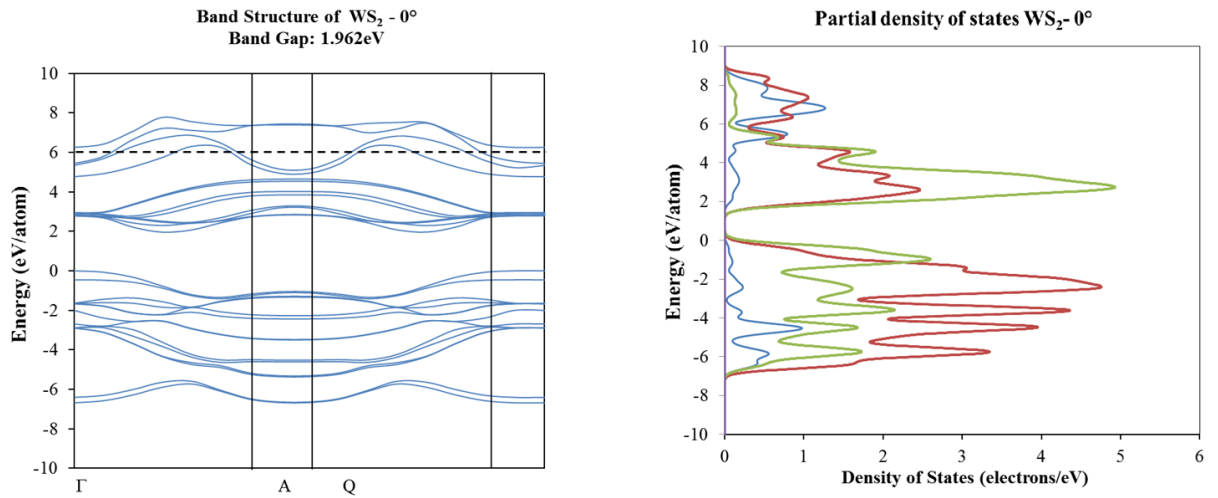

Figure 7. Cont. 

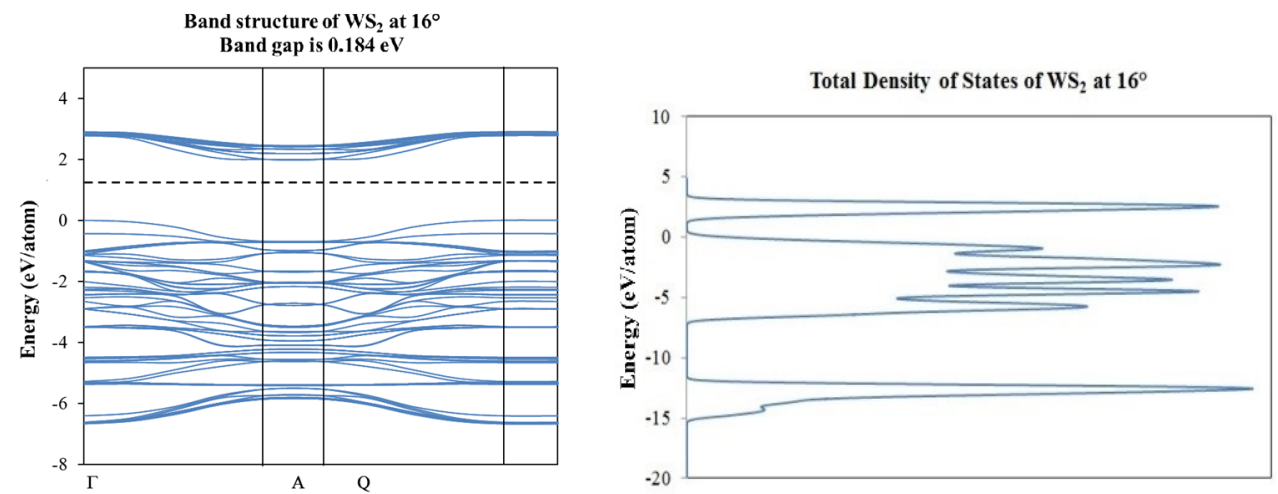

Figure 7. Band structure and partial total density of states plots corresponding to $0^{\circ}$ (top) and $16^{\circ}$ (bottom) of rotation in $\mathrm{WS}_{2}$ molecular structure.
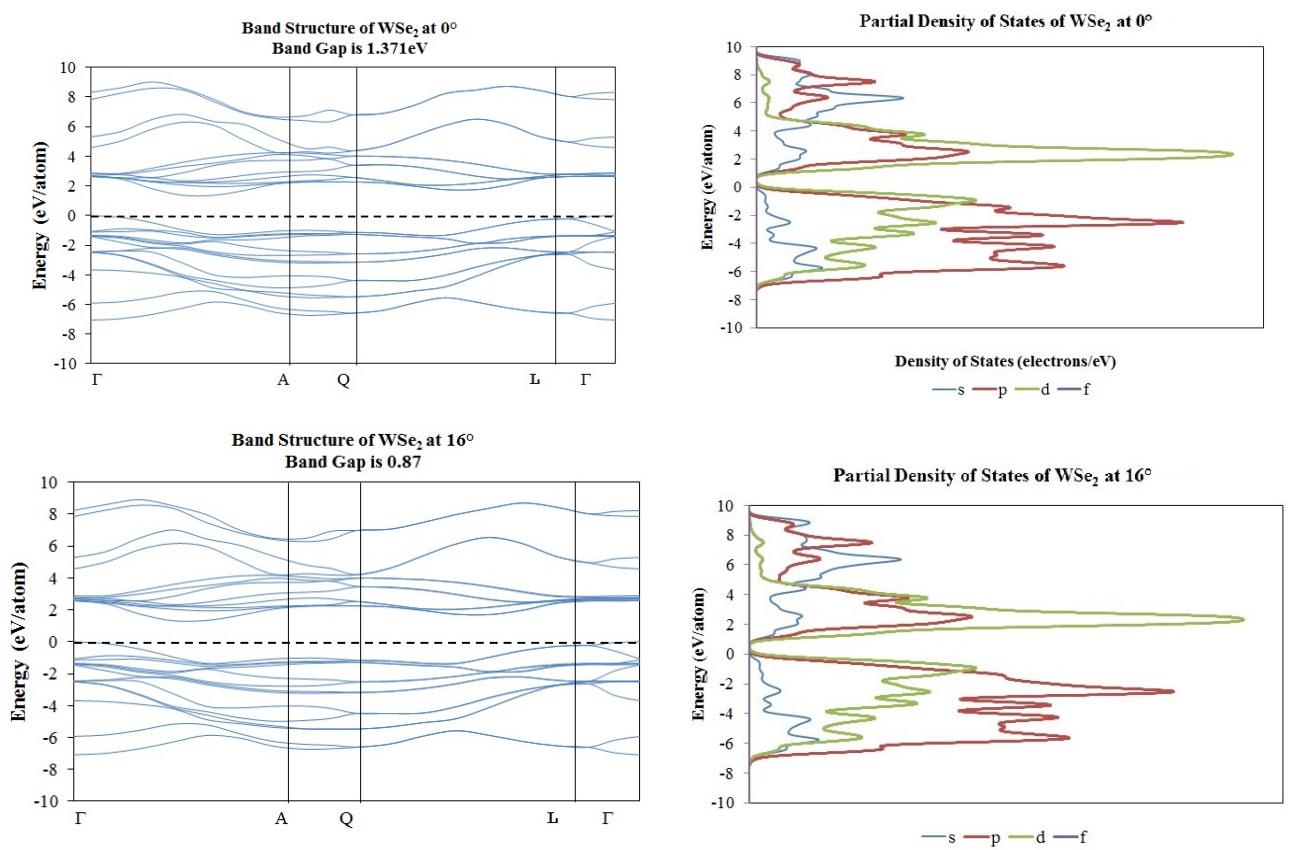

Figure 8. Band structure and partial density of states plots corresponding to $0^{\circ}$ (top) and $16^{\circ}$ (bottom) of rotation in $\mathrm{WSe}_{2}$ molecular structure.

Because of the large data calculations, all band gap values were added and plotted with the degree of rotation, as presented in Figure 9 in band gap vs. degrees of rotation. It is possible to achieve appropriate conclusions by using this fast and low-cost approach helps understand Bollman O-lattice formation due to the "twisting" of two-layered molecular structures, subjected to numerical density functional theory computations. It is possible to achieve high accuracy, as reported before [9], a high-throughput approximation was obtained using the CASTEP algorithm to describe the electronic structure transitions in layered molecular nanostructure materials. 


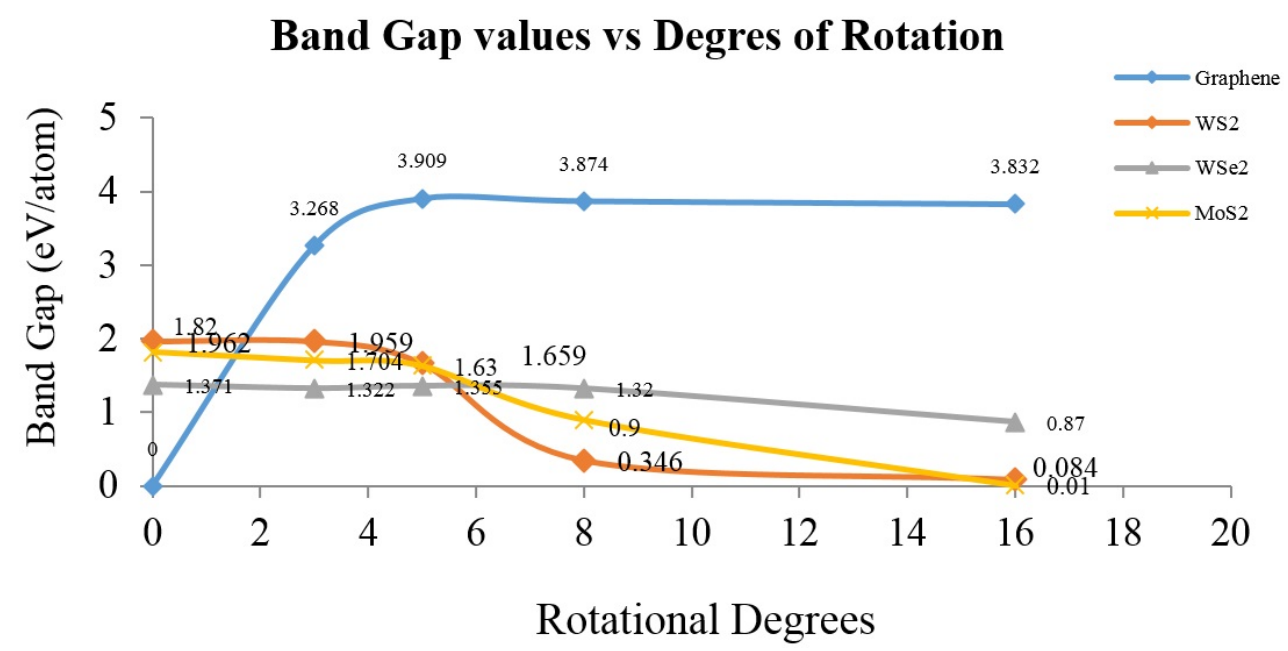

Figure 9. Band gap values of layered molecular models corresponding to graphene, $\mathrm{WS}_{2}, \mathrm{WSe}_{2}$ and $\mathrm{MoS}_{2}$, as obtained from DFT numerical simulations using CASTEP algorithm.

\section{Computational Details}

\subsection{Multislice HRTEM Simulations}

Computer-assisted transmission electron microscope simulations were completed using a fully dynamical calculation multi-slice method using projected potential:

$$
f(U)=\sum_{i=1}^{n} a_{i} e^{\left(-b_{i} U^{2}\right)}
$$

where $U$ represents the coordinates in reciprocal space $(u, v, w)$ as described by Gomez-Rodríguez [15], projecting the potential over the (001) $c$-axis and adjusting $a$ and $b$ coefficients in a range of $98<a<$ 128 and $96<b<128$ to obtain the optimal conditions in comparison to some experimental HRTEM results, as presented in the literature [3,7]. The crystallographic structures were built using a graphical user interface as presented in the builder module of the Accelrys 6.1-Materials Studio ${ }^{\circledR}$ package with crystallographic parameters as found from Joint Committee on Powder Diffraction Standards (JCPDS) data base. All honeycomb structures were created when exerting mechanical rotations at $3^{\circ}, 5^{\circ}, 8^{\circ}$, and $16^{\circ}$, with respect to the (001) c-basal axis, always keeping an inter-layer distance of $6.20 \AA$ from molybdenum to molybdenum (Mo-Mo) in $\mathrm{MoS}_{2}, 6.4 \AA$ from tungsten to tungsten $(\mathrm{W}-\mathrm{W})$ in $\mathrm{WS}_{2}$ and $6.7 \AA$ in $\mathrm{WSe}_{2}$ and $3.4 \AA$ in carbon to carbon as found in carbonic rings of graphene layers.

\subsection{Density Functional Theory Calculations}

The electronic structure of each strain's O-lattice was calculated using a seed from two large layers as built in the Materials Studio 6.1 graphical user interface; each superlattice (O-lattice) consisted of 1804 atoms approximately, as shown in Figure 1. Thus, to achieve fast conversion criteria and to reduce the computational time, the electronic calculations were performed using a representative portion called "seed", consisting of 12 to 24 atoms, as shown in the inset of Figure 1. The "seed" was placed in a P1 (no symmetry) crystal and with varying lattice parameters from $16.0 \AA \leq a \leq 18.0 \AA, 17.0 \AA \leq b \leq$ $21.0 \AA, 15.0 \leq c \leq 17.0 \AA$ and crystal lattice angles of $\alpha=\beta=90^{\circ}$ and $\gamma=120^{\circ}$. These particular distances were selected cautiously to avoid interactions with neighboring atoms in periodic cell conditions, which could yield incorrect results in both the band structure and density of states calculations. Before calculating the density of states and band structure calculations, both untwisted and twisted "seeds" were subjected to geometrical optimizations using the Broyden-Fletcher-Goldfarb-Shanno algorithm, as described by Fischer and Almlöf [16]. All DFT computations were performed using Cambridge 
Serial Total Energy Package (CASTEP) with a revised Perdew-Burke-Ernzerhof functional [17] general gradient approximation, a cutoff energy of $300 \mathrm{eV}$ in the reciprocal space for the gamma point only with a self-consistent field (SCF) convergence threshold of $1 \times 10^{-6} \mathrm{eV}$ per atom and without any thermal smearing.

\section{Conclusions}

From previous investigations it is possible to achieve observations of the $\mathrm{MoS}_{2}$ stacked structure using experimental HRTEM; then, using dynamical multi-slice HRTEM simulations it was possible to determine a structural meaning of those honeycomb-like observations. Here we presented a more extensive study including graphene, $\mathrm{WS}_{2}$ and $\mathrm{WSe}_{2}$ layered materials, observing a new concept as presented by Bollman corresponding to the formation of a new lattice, the so-called O-lattice. To further understand the electronic structure nature of this new O-lattice, we proceeded with DFT numerical simulations where it was possible to determine some transitions and band gap reductions due to strain caused by rotating the lattices as presented here; the undergoing mechanism of those electronic states can be fully understand if a combined experiment is set by using Extended X-ray Absorption Fine Structure (EXAFS) on exfoliated 2D layered materials and with of computer assisted X-ray simulations, since we believe a crystallographic transition is clearly occurring, which can cause a space group change from hexagonal into trigonal, perturbing d-orbital clouds between layers as discussed briefly in Reference [9].

Acknowledgments: Instituto de Ingeniería y Tecnología de Universidad Autónoma de Cd. Juárez and University of Texas at El Paso for usage of computational resources. Javier Polanco-Gonzalez thank the Consejo Nacional de Ciencia y Tecnología (CONACyT-México) for financial support on repatriation fellowship \#262748.

Author Contributions: Javier Polanco-Gonzalez and Jesús Alfredo Carranco-Rodríguez were in charge of computer-assisted numerical simulations by DFT methods. Manuel Ramos was in charge of computer-assisted transmission electron images. Pierre G. Mani-Gonzalez and Jose L. Enríquez-Carrejo were in charge of data processing and interpretation. The manuscript was completed and written by Manuel Ramos, José L. Enríquez-Carrejo and Javier Polanco-Gonzalez.

Conflicts of Interest: The authors declare no conflict of interest.

\section{References}

1. Weisser, O.; Landa, S. Sulfide Catalysts, Their Properties and Applications; Pergamon Press: New York, NY, USA, 1973.

2. Perea-López, N.; Lin, Z.; Pradhan, N.R.; Iñiguez-Rábago, A.; Elías, A.L.; McCreary, A.; Lou, J.; Ajayan, P.M.; Terrones, H.; Balicas, L.; et al. CVD-grown monolayered $\mathrm{MoS}_{2}$ as an effective photosensor operating at low-voltage. 2D Mater. 2014, 1, 011004. [CrossRef]

3. Chianelli, R.R.; Ruppert, A.F.; Jose, M.; Va, A. HREM studies of layered transition metal sulfide catalytic materials. Catal. Today 1995, 23, 269-281. [CrossRef]

4. Vazquez, A. High Resolution electron microscopy of $\mathrm{MoS}_{2}: \mathrm{Ni}, \mathrm{MoS}_{2}: \mathrm{Co}_{\text {and }} \mathrm{MoS}_{2}: \mathrm{Fe}$ layered crystals. Mater. Lett. 1998, 35, 22-27. [CrossRef]

5. Kobayashi, K. Moiré patterns in scanning tunneling microscopy images of layered materials. J. Vac. Sci. Technol. B 1996, 14, 175-178. [CrossRef]

6. Jellinek, F.; Brauer, G.; Müller, H. Molybdenum and Niobium Sulphides. Nature 1960, 185, 376-377. [CrossRef]

7. Reyes-Gasga, J.; Tehuacanero, S.; Yacamán, M.J. Moiré patterns in high resolution electron microscopy images of $\mathrm{MoS}_{2}$. Microsc. Res. Tech. 1998, 40, 2-9. [CrossRef]

8. Jasinski, J.B.; Dumpala, S.; Sumanasekera, G.U.; Sunkara, M.K.; Ouseph, P.J. Observation and interpretation of adjacent Moire patterns of different shapes in bilayer graphene. Appl. Phys. Lett. 2011, 99, 073104. [CrossRef]

9. Ramos, M.A.; Chianelli, R.; Enriquez-Carrejo, J.L.; Gonzalez, G.A.; Berhault, G. Angular depdence of metallic states in $2 \mathrm{H}-\mathrm{MoS}_{2}$ slabs. Comput. Mater. Sci. 2014, 84, 18-22. [CrossRef]

10. Bollman, W. Crysrtal Defects And Crystalline Interfaces; Springer: Berlin, Germany, 1970. 
11. Remskar, M.; Mrzel, A.; Skraba, Z.; Jesih, A.; Ceh, M.; Demšar, J.; Stadelmann, P.; Lévy, F.; Mihailovic, D. Self-Assembly of Subnanometer-Diameter Single-Wall MoS 2 Nanotubes. Science 2001, 292, 479-481. [CrossRef] [PubMed]

12. Gómez Rodríguez, A.; Romeu Casajuana, D. Reciprocity relations for Bollmann's O-lattice. Rev. Mex. Fís. 2007, 53, 139-143.

13. Novoselov, K.S.; Geim, A.K.; Morozov, S.V.; Jiang, D.; Zhang, Y.; Dubonos, S.V.; Grigorieva, I.V.; Firsov, A.A. Electric field effect in atomically thin carbon films. Science 2004, 306, 666-669. [CrossRef] [PubMed]

14. Zhang, Y.; Tang, T.T.; Girit, C.; Hao, Z.; Martin, M.C.; Zettl, A.; Crommie, M.F.; Shen, R.Y.; Wang, F. Direct observation of a widely tunable bandgap in bilayer graphene. Nat. Lett. 2009, 459, 820-823. [CrossRef] [PubMed]

15. Gómez-Rodríguez, A.; Beltrán-del-Río, L.M.; Herrera-Becerra, R. SimulaTEM: Multislice simulations for general objects. Ultramicroscopy 2010, 110, 95-104. [CrossRef] [PubMed]

16. Fischer, T.H.; Almlöf, J. General methods for geometry and wave function optimization. J. Phys. Chem. 1992, 96, 9768-9774. [CrossRef]

17. Perdew, J.P.; Burke, K.; Ernzerhof, M. Generalized Gradient Approximation Made Simple. Phys. Rev. Lett. 1996, 77, 3865. [CrossRef] [PubMed]

(C) 2017 by the authors; licensee MDPI, Basel, Switzerland. This article is an open access article distributed under the terms and conditions of the Creative Commons Attribution (CC BY) license (http:/ / creativecommons.org/licenses/by/4.0/). 